\title{
3D Characterization of Diffusivities and Its Impact on Mass Flux and Concentration Overpotential in SOFC Anode
}

\author{
Xuekun Lu, ${ }^{a}$ Benhard Tjaden, ${ }^{a, *}$ Antonio Bertei, ${ }^{\mathrm{b}}$ Tao Li, ${ }^{\mathrm{c}}$ Kang Li, ${ }^{\mathrm{c}}$ Dan Brett, ${ }^{\mathrm{a}}$ \\ and Paul Shearing ${ }^{a, z}$ \\ ${ }^{a}$ Electrochemical Innovation Lab, Department of Chemical Engineering, University College London, \\ London WC1E 7JE, United Kingdom \\ ${ }^{b}$ Department of Earth Science and Engineering, Imperial College London, London SW7 2AZ, United Kingdom \\ ${ }^{c}$ Department of Chemical Engineering, Imperial College London, London SW7 2AZ, United Kingdom
}

\begin{abstract}
In recent years great effort has been taken to understand the effect of gas transport on the performance of electrochemical devices. This study aims to characterize the diffusion regimes and the possible inaccuracies of the mass transport calculation in Solid Oxide Fuel Cell (SOFC) anodes when a volume-averaged pore diameter is used. 3D pore size distribution is measured based on the extracted pore phase from an X-ray CT scan, which is further used for the calculation of a Knudsen number $\left(K_{n}\right)$ map in the porous medium, followed by the voxel-based distribution of the effective diffusion coefficients for different fuel gases. Diffusion fluxes in a binary gas mixture using the lower boundary, upper boundary and average effective coefficients are compared, and the impact on overpotential is analyzed. The results show that pore diameters from tens to hundreds of nanometers result in a broad range of Knudsen number (1.1 $\sim 4.8$ and $0.6 \sim 3$ for $\mathrm{H}_{2}$ and $\mathrm{CH}_{4}$ respectively), indicative of the transitional diffusion regime. The results highlight that for a porous material, such as an SOFC anode where Knudsen effects are non-negligible, using a volume-averaged pore size can overestimate the mass flux by $\pm 200 \%$ compared to the actual value. The characteristic pore size should be chosen sensibly in order to improve the reliability of the mass transport and electrochemical performance evaluation.

(C) The Author(s) 2017. Published by ECS. This is an open access article distributed under the terms of the Creative Commons Attribution Non-Commercial No Derivatives 4.0 License (CC BY-NC-ND, http://creativecommons.org/licenses/by-nc-nd/4.0/), which permits non-commercial reuse, distribution, and reproduction in any medium, provided the original work is not changed in any way and is properly cited. For permission for commercial reuse, please email: oa@electrochem.org. [DOI: 10.1149/2.0111704jes] All rights reserved.

(cc) BY-NC-ND
\end{abstract}

Manuscript submitted November 7, 2016; revised manuscript received January 6, 2017. Published January 21, 2017.

Mass transport can significantly limit the reaction rate and lead to concentration polarization in electrochemical devices such as fuel cells, electrolysers and oxygen transport membranes, especially under the conditions of high operating current density and fuel conversion ratios. ${ }^{1}$ Besides the material-specific transport properties of chemical species (e.g., diffusivity, viscosity, etc.), mass transport is mainly dependent on microstructural parameters of the electrode such as porosity, tortuosity and pore size..$^{2-4}$ In recent years great effort has been taken to understand the effect of gas transport on the performance of electrochemical devices in terms of electrochemical impedance, ${ }^{5}$ concentration polarization loss, ${ }^{4,6}$ and electrochemical simulation. ${ }^{7-10}$

Concentration polarization is caused by the consumption of the fuel gas resulting in a reduction of concentration at the anode/electrolyte interface. In solid oxide fuel cell (SOFC) anodes, concentration polarization is governed by the diffusion of fuel gas (e.g. $\left.\mathrm{H}_{2}\right)$ from the gas inlet to the triple phase boundary. A faster diffusion rate can contribute to a larger partial pressure of the fuel gas within the pores and thus a larger limiting current density, which in turn mitigates the polarization loss. ${ }^{11}$

The effective diffusivity is closely related to the temperature, pressure, gas species as well as to the microstructure of the porous material. ${ }^{12}$ A considerable amount of work has been reported to measure the diffusivity, such as the experimental methods including diffusion cells, ${ }^{13}$ gas chromatography, ${ }^{14}$ thermo-gravimetric analysis, ${ }^{15}$ as well as theoretical calculations based on the continuum physics, such as the binary diffusivity using Chapman-Enskog theory, the Knudsen diffusion coefficient and the effective diffusion coefficient. ${ }^{16}$ The mechanisms of the mass transport are closely related to the pore size that partly determines the Knudsen number $K_{n}$, which will be discussed in the next section. Pore size measurements are often based on the analysis of $2 \mathrm{D}$ cross-section images obtained by scanning electron microscopy. ${ }^{17}$ However, this approach requires correction factors based on stereology, ${ }^{18}$ and a large number of observations must be made for a statistically reliable result. This method is unable to display the pore size spatial distribution and cannot account for percolation. Pore size can also be measured via porosimeters using the

*Electrochemical Society Student Member.

${ }^{z}$ E-mail: p.shearing@ucl.ac.uk bubble point method, ${ }^{19}$ but again, this method only gives a spatially averaged characteristic pore size for the material measured. In most cases, a mean pore diameter is used as the characteristic pore size for the diffusivity calculation. However, in porous support materials, such as the SOFC anode, local pore size phase varies from tens of nanometers to microns, and gas species diffuse selectively in the porous path depending on their mean free path and the pore size, tortuosity and constrictivity. Thus the assumption of uniform pore size can lead to overestimation of the diffusive flux, ${ }^{20}$ and corresponding underestimation of the tortuosity. ${ }^{13}$

$\mathrm{X}$-ray computed tomography $(\mathrm{CT})$ is a widely used technique to non-destructively inspect the 3D micro-structure of a material based on different X-ray absorption coefficients. ${ }^{21,22}$ A considerable amount of work has been reported to measure the pore size distributions, porosity and tortuosity in porous support materials such as $\mathrm{SOFC}^{23}$ and batteries. ${ }^{24,25}$ The reconstructed sample volume can be subsequently used as a real model for the image-based simulation of porous phase tortuosity, ${ }^{26,27}$ diffusion flux ${ }^{16}$ and electrochemical performance. ${ }^{9}$

This study aims to characterize the diffusion regimes in an SOFC anode for different fuel gases using the X-ray nano-CT technique, followed by the quantitative evaluation of the mass transport phenomenon in a binary gas mixture based on a broad distribution of the voxel-basis effective diffusion coefficients. These will provide with insights in the effect of mean pore diameter on the diffusive mass flux and overpotential calculation.

\section{Theory}

Transport of gas molecules in porous media involves molecular interactions between gas molecules as well as the collisions between gas molecules and the walls of the porous medium. ${ }^{2}$ There are three possible transport mechanisms: molecular diffusion, Knudsen diffusion and viscous flow, and the predominant mechanism in the transport regime will depend on the gas species, temperature, pressure and microstructure. ${ }^{12,28}$ Molecular diffusion refers to the relative motion and collisions between different gas species; Knudsen diffusion covers the effect of collisions between gas molecules and the walls of the porous medium, and viscous flow is associated with the bulk flow of the gas stream due to a pressure gradient. When the gas stream 
Table I. Molecular parameters and the corresponding mean free path for $\mathrm{H}_{2}$ and $\mathrm{CH}_{4}$.

\begin{tabular}{ccccc}
\multicolumn{2}{c}{$\begin{array}{c}\text { Molecular } \\
\text { diameter }(\AA)\end{array}$} & \multicolumn{3}{c}{ Mean free path $\lambda$ in $(\mathrm{nm})$ at $p=1 \mathrm{~atm}$} \\
\cline { 3 - 5 } & $600^{\circ} \mathrm{C}$ & $800^{\circ} \mathrm{C}$ & $1000^{\circ} \mathrm{C}$ \\
\hline $\mathrm{H}_{2}$ & 2.74 & 351 & 432 & 513 \\
$\mathrm{CH}_{4}$ & 4.14 & 153 & 187 & 223
\end{tabular}

is stagnant (i.e. no viscous flow), the Knudsen number $\left(K_{n}\right)$ is often calculated as an indication of the governing mechanism in the gas diffusion regime,

$$
K_{n}=\frac{\lambda}{d_{p}}
$$

where $d_{p}$ is the pore size, and $\lambda$ is the gas mean free path, which can be obtained by

$$
\lambda=\frac{K_{b} T}{\sqrt{2} p \pi d_{g}^{2}}
$$

where $K_{b}$ is the Boltzmann constant $\left(1.3807 \times 10^{-23} \mathrm{~J} / \mathrm{K}\right), T$ is the temperature, $p$ is the pressure, and $d_{g}$ is the characteristic diameter of the gas molecule, which can be estimated using covalent and van der Waals radii.

If $K_{n}<0.1$, the molecular diffusion is dominant and the Knudsen diffusion can be neglected; if $K_{n}>10$, the effect of Knudsen diffusion outweighs the molecular diffusion because of the frequent collisions between the molecules and the porous medium. As for $0.1<K_{n}<10$, the so-called transition diffusion regime, diffusion is governed by both mechanisms. Previous works suggest that $K_{n}$ is in the range of $0.1<K_{n}$ $<5$ for most of the SOFC electrodes, ${ }^{29}$ however the distribution of the local pore size is often neglected and an average pore size for the whole micro-structure is used. Literatures have recorded different concepts and details of the 3D pore size distribution (PSD) measurement. ${ }^{30,31}$ Accordingly, a great amount of work assumes a uniform $K_{n}$ in the diffusion regime. ${ }^{6,20}$ The molecular parameters and the calculated mean free path $\lambda$ are summarized in Table I.

Knudsen diffusion can be investigated either using high-fidelity numerical methods such as Direct Simulation Monte Carlo (DSMC) and Lattice-Boltzmann (LB), ${ }^{33-35}$ or using continuum methods such as Fick's law, Maxwell-Stefan Model and Dusty Gas Model. ${ }^{4,10,36}$ Numerical methods have the advantages over continuum methods in terms of more flexible definition of gas-wall interactions, which are not explicitly modelled in this study. However, the problem of boundary conditions in Knudsen regime remains for LBM because the pore wall morphology is voxelized, which hinders proper handling of redirection collisions as reported by Berson et al. ${ }^{37}$

In binary mixtures, Fick's law (FL) is the most widely used diffusion model to calculate the diffusion flux of molecular diffusion due to its simplicity. ${ }^{38}$ This study evaluates the diffusion on mass flux basis, but it is also applicable to mole flux basis. ${ }^{36}$ Fick's law correlates the diffusion flux with the concentration gradient under steady-state as

$$
J_{i}=-D_{e f f} \nabla\left(c_{i}\right) \quad(i=1,2,3 \ldots)
$$

where $D_{\text {eff }}$ is the effective diffusion coefficient (discuss later), $c_{i}$ is the mass concentration of the gas species $i, J_{i}$ is the mass flux of the gas species $i$.

The binary diffusion coefficient $D_{i j}{ }^{39,40}$ is defined as $\left(\mathrm{cm}^{2} / \mathrm{s}\right)$

$$
D_{i j}=\frac{10^{-3} T^{1.75}}{p\left(V_{i}^{1 / 3}+V_{j}^{1 / 3}\right)^{2}}\left\{\frac{1}{M_{i}}+\frac{1}{M_{j}}\right\}^{0.5}
$$

where $T$ is the temperature $(K), p$ is the pressure $(\mathrm{atm}), M_{i}$ and $M_{j}$ are the molar mass of the two gas species $(\mathrm{g} / \mathrm{mol}), V_{i}$ and $V_{j}$ are the Fuller diffusion volume for gas species $i$ and $j$ respectively: $\mathrm{H}_{2}$ (7.07), $\mathrm{CH}_{4}$ (24.5), $\mathrm{N}_{2}(17.9)$ and $\mathrm{H}_{2} \mathrm{O}(12.7) .{ }^{39}$
Knudsen diffusivity becomes important if the mean free path of the gas molecules is of the same order of magnitude or even larger than the pore diameter. The Knudsen diffusivity of gas species can be estimated by ${ }^{41}$

$$
D_{i}^{k}=\frac{d_{p}}{3} \sqrt{\frac{8 R T}{\pi M_{i}}}
$$

where $R$ is the ideal gas constant. When both molecular diffusion and Knudsen diffusion are comparable, an effective diffusivity is employed using the Bosanquet equation: ${ }^{41,42}$

$$
\frac{1}{D_{e f f}}=\frac{1}{D_{i j}}+\frac{1}{D_{i}^{k}}
$$

The use of the Bosanquet equation to solve the gas transport problem at the microscopic pore-resolved scale, where pore dimensions are comparable with mean-free path lengths, is well validated by Krishna et al., ${ }^{42}$ who confirm that there is a good agreement between the diffusivity simulated by Molecular Dynamics and Bosanquet methods especially if the gas species is poorly adsorbing on the pore wall, such as $\mathrm{H}_{2}$.

\section{Experimental}

Materials.-Commercially available powders of yttria-stabilized zirconia (YSZ8, surface area $35.5 \mathrm{~m}^{2} \mathrm{~g}^{-1}$, mean particle size (d50) $0.1-0.4 \mu \mathrm{m})$ and nickel oxide $\left(\mathrm{NiO}\right.$, surface area $3.7 \mathrm{~m}^{2} \mathrm{~g}^{-1}$, mean particle size (d50) 0.5-1.5 $\mu \mathrm{m}$ ) were purchased from Inframat Advanced Materials and used as supplied. Polyethersulfone (PESf) (Radal A300, Ameco Performance, USA), 30-dipolyhydroxystearate (Arlacel P135, Uniqema), and N-methyl-2-pyrrolidone (NMP, HPLC grade, VWR) were used as the polymer binder, dispersant and solvent, respectively.

A suspension composed of ceramic particles (wt\%): YSZ (25.6), $\mathrm{NiO}$ (38.4), DMSO (28.4), PESf (7.1), Arlacel P135 (0.5), together with solvent and polymer binder were mixed for 3-4 days via planetary ball milling (263 rpm, SFM-1 Desk-top Miller, MTI Corporation, USA) to obtain homogeneity. Prior to the fabrication, the suspension was degassed under vacuum to fully eliminate air bubbles trapped inside. The tubular anode support was fabricated via a phase inversion assisted extrusion process. ${ }^{43-47} \mathrm{~A}$ sintering step was then undertaken at $1450^{\circ} \mathrm{C}$ for 6 hours to convert the precursors into rigid ceramic body for subsequent characterization. A reduction step at $700^{\circ} \mathrm{C}$ for 2.5 hours under pure hydrogen atmosphere was conducted to convert $\mathrm{NiO}$ into $\mathrm{Ni}$ before the X-ray CT scan. A small sample $(14 \times 14 \times$ $12 \mu \mathrm{m}^{3}$ ) was extracted from the reduced anode and fit into the field of view in the X-ray nano-CT scan. More details on sample fabrication can be found from the work by T. Li. ${ }^{48}$

X-ray computed tomography (CT).-Non-destructive X-ray nano-computed tomography (CT) was undertaken on a Zeiss Xradia Ultra $810 \mathrm{X}$-ray microscope at the Electrochemical Innovation Lab (EIL), University College London. This machine produces a $5.4 \mathrm{keV}$ quasi-monochromatric parallel beam by the use of X-ray optics. A small piece of sample was extracted from the anode in order to obtain a cylindrical imaged volume with $16 \mu \mathrm{m}$ diameter, giving a voxel size of $32 \mathrm{~nm}$. A projection was collected every 60 seconds using a 1024 by 1024 pixel 16-bit camera under binning 2 mode, each projection separated by an angle of $0.15^{\circ}$. This resulted in 1201 projections over a full scan, which were then reconstructed by a standard filtered back projection algorithm in the Zeiss XMReconstructor software. The reconstructed volume was subsequently imported into the image processing software Avizo 9.0 (Visualization Sciences Group, Bordeaux) for porous phase segmentation using watershed algorithm.

Pore diameter measurement.-The pore diameter 3D distribution of the scanned samples was evaluated using the ImageJ (Abramoff et al., 2007) plugin BoneJ. ${ }^{49}$ This software achieves the pore size distribution by computing the Euclidean distance transformation of 


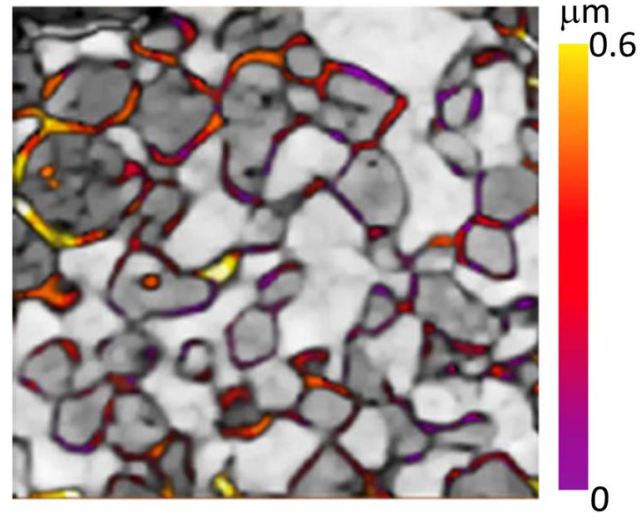

Figure 1. Local pore diameter distribution of the SOFC anode in a representative 2D planar slice of the gray scale image. The dark and light gray phases correspond to $\mathrm{Ni}$ and $\mathrm{YSZ}$ respectively.

the imported pore phase and extracting the distance ridge, followed by the fitting of the largest spheres locally at each point on the ridge to obtain the local diameter. ${ }^{49,50}$ Fig. 1 shows the local pore size distribution on a planar slice extracted from the reconstructed volume of the porous anode. The color scale represents the local pore diameter in $3 \mathrm{D}$. The resulting pore diameter distribution was then exported to MATLAB (Mathworks Inc.) as an 8-bit volume in the form of a .rawfile, where it was further processed to calculate local Knudsen numbers and diffusion coefficients. The pore diameter measured by image processing is compared to the experimental method using mercury intrusion porosimetry (Micromeritics AutoporeIV).

Mass flux simulation.-The segmented porous phase structure from the reconstructed $3 \mathrm{D}$ volume of the sample was saved as a surface mesh (ASCII *.stl) file using Avizo and subsequently was imported into the computational fluid dynamics (CFD) software Star$\mathrm{CCM}+(\mathrm{CD}$-Adapco Inc., London). The geometry of the porous phase is shown in Fig. 2a. The surface mesh generated by Avizo appears to be triangular shape (Fig. 2b), which was re-meshed by built-in remesher tool in Star-CCM+ to ensure that the mesh is closed and manifold, with no free edge and holes. The re-meshing procedure is monitored so that the volume change of the porous phase is below $1 \%$. Finally an adaptive polyhedral volume mesh was generated and is seen in Fig. 2c. A three-dimensional steady-state mass-conservation of $\mathrm{H}_{2}$ is solved, formulating as the divergence of the mass flux vector (i.e. $\nabla \cdot J_{H_{2}}=0$ ), with $\mathrm{H}_{2} \mathrm{O}$ balanced locally. The mass flux Knudsen diffusion is approximated using continuum physics, assuming zero flux condition normal to the interior pore walls (i.e., $\boldsymbol{n} \cdot J_{H_{2}}=0, \boldsymbol{n}$ is the unit normal vector). Dirichlet boundary conditions are applied at the inlet and outlet. The modelling parameters and conditions used in this study are summarized in Table II.

\section{Results and Discussion}

Pore diameter measurement: CT analysis vs. mercury intrusion porosimetry.-The reconstructed volume of the anode is shown in

Table II. CFD simulation parameters and boundary conditions for the $\mathrm{H}_{2}$ diffusion in a binary mixture with $\mathrm{H}_{2} \mathrm{O}$.

Mesh type

Mesh number

Pressure (atm)

Temperature $\left({ }^{\circ} \mathrm{C}\right)$

Gas inlet boundary

Gas outlet boundary

Sample dimension $\left(\mu \mathrm{m}^{3}\right)$
Polyhedral volume mesh

$1,170,000$

1.01

1000

$100 \% \mathrm{H}_{2}$

$0 \% \mathrm{H}_{2}$

$9 \times 6 \times 4$

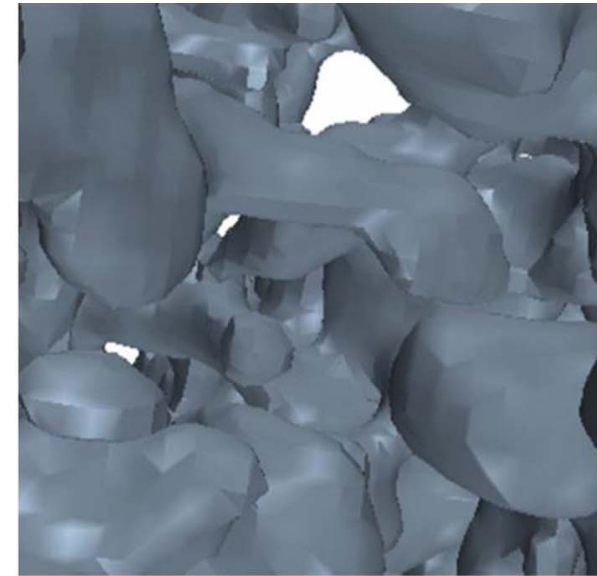

(a)

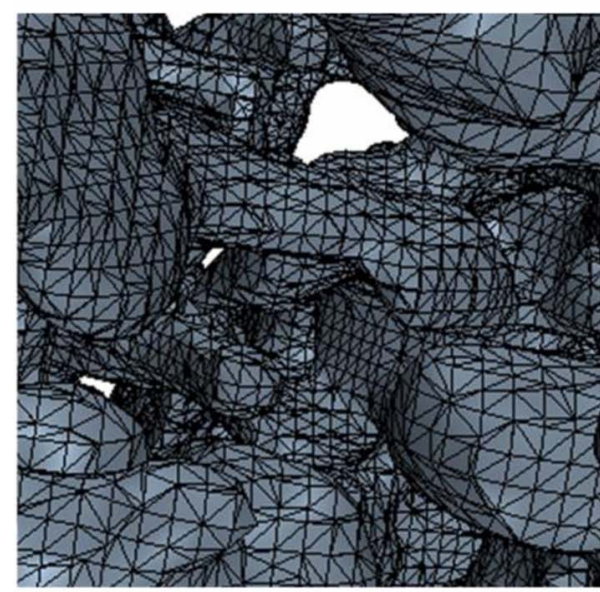

(b)

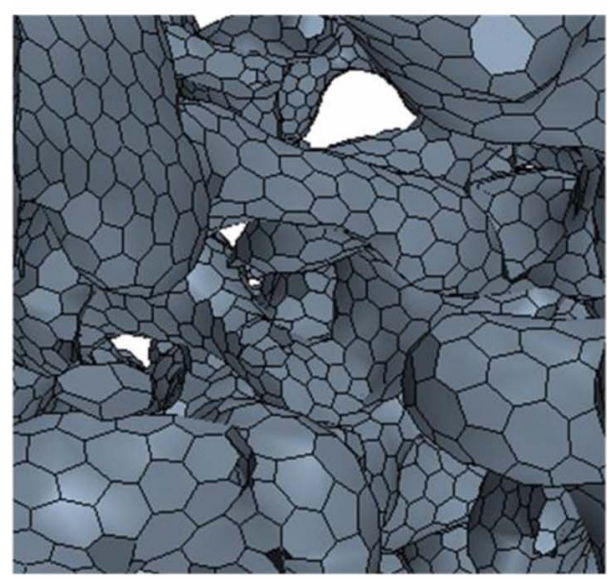

(c)

Figure 2. (a) Geometry of the porous phase in the anode (b) triangular surface mesh generated by Avizo (c) polyhedral volume mesh generated by $\mathrm{Star}-\mathrm{CCM}+$

Fig. 3. A spongy porous phase is observed to be surrounded by the solid phase. By thresholding the grayscale volume, the porous phase can be extracted and rendered in 3D. The segmented porous phase was then skeletonized and represented with the colormap indicating the local size variation of the pore channels. The mean pore diameter is measured to be $0.16 \pm 0.05 \mu \mathrm{m}$. 

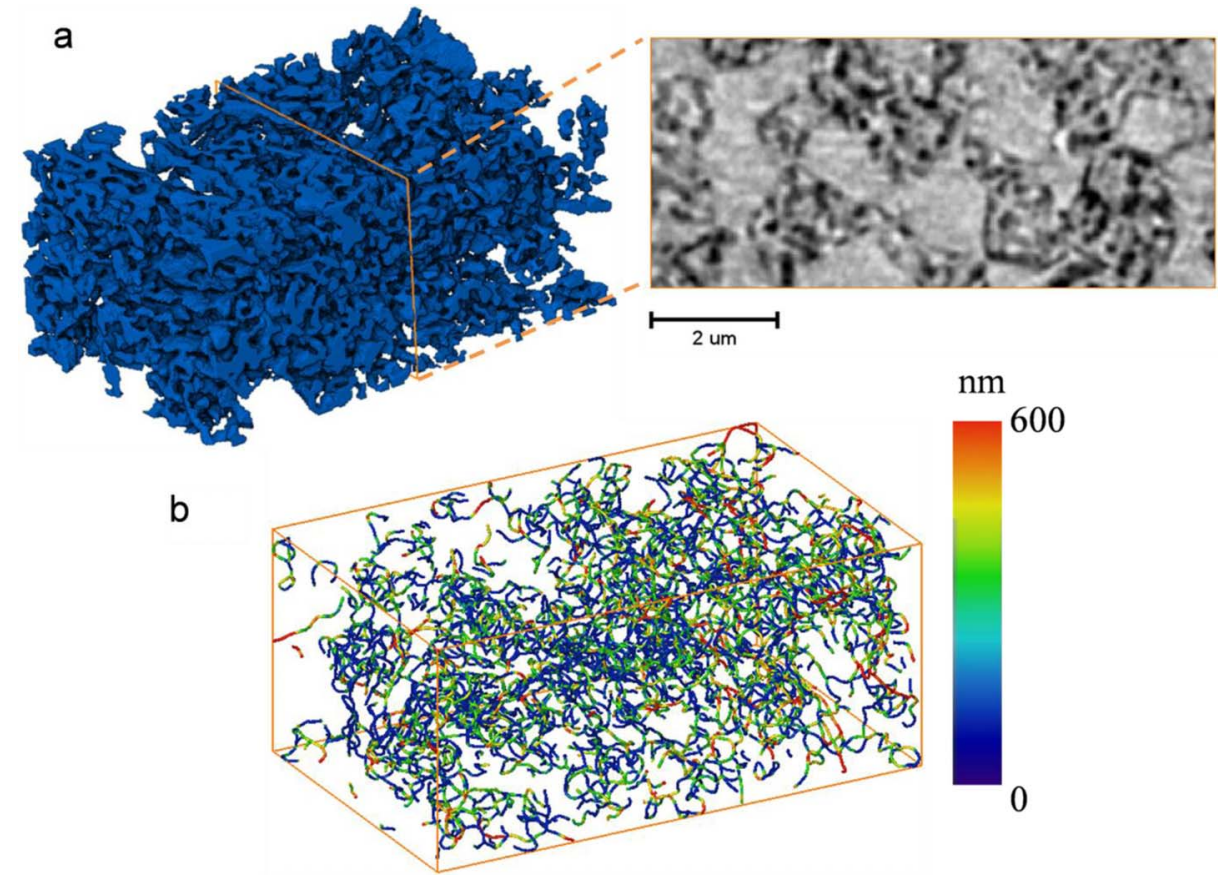

$\mathrm{nm}$

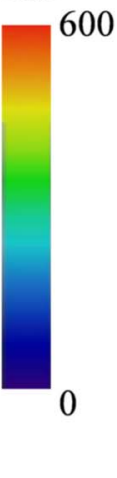

Figure 3. (a) Virtual slice of the reconstructed anode represented as grayscale image, shown alongside with the segmented and rendered porous phase in 3D; (b) Skeletonization of the pore network with the colormap indicating local pore diameter.
Mercury intrusion porosimetry was conducted on an intact novelstructured tubular SOFC anode, where the spongy sample was extracted from. The result is shown in Fig. 4a, alongside with the pore size distribution (Fig. 4b) obtained by CT analysis as a comparison. Two peaks are observed from experiment measurement: the LHS peak represents the pore size in the spongy region and the RHS peak corresponds to the pore size at the entrance of the micro-channels. In this study we focus only on the pore size distribution of the spongy region.

It is found that the pore diameter measured by mercury intrusion is slightly larger than the image processing method. However, this disparity is insignificant considering the variation of the mean pore size distribution on different samples using image processing $(0.16 \pm$ $0.05 \mu \mathrm{m}$ ) as mercury intrusion method covers the pore size distribution over the entire anode, in contrast with the image processing method, which includes only a small volume. But a small volume from X-ray $\mathrm{CT}$ contributes to a higher resolution in order to capture the smallest pores (approx. $50 \mathrm{~nm}$ ) as is seen from Fig. 4b, which is beyond the capability of mercury intrusion porosimetry. Thus, it is important to note that the macroscopic methods can overestimate the mass flux by neglecting the smallest pore size in the porous volume, although the average pore size is identical to that of CT analysis.

Representative volume analysis (RVA).- - The RVA is conducted using an open-source Matlab application Taufactor ${ }^{51}$ to plot the variation of microstructure parameters against increments of the sample volume to investigate the extent to which the volume can be considered representative of the material in general. Three methods are used to study the change of porosity related to the sample volume: IsoDilation (growing a cuboid from the center isotropically), ConstHeight (growing the hexahedron with fixed height) and ConstArea (with constant cross section area). The tortuosity factor vs. volume fraction is also examined using ConstArea to study the representative distance of the diffusion domain in this material. The schematics and the results are shown in Fig. 5. Results show that the porosities measured by all three methods converge at $18 \%$ when approx. $80 \%$ of the sample volume are included, which corresponds to $160 \mu \mathrm{m}^{3}$. Moreover, the tortuosity factor also converges at a stable level at half of the total diffusion distance used in this study. This evidence proves the validity and representativeness of the pore diameter measurement and mass flux simulation in this study.

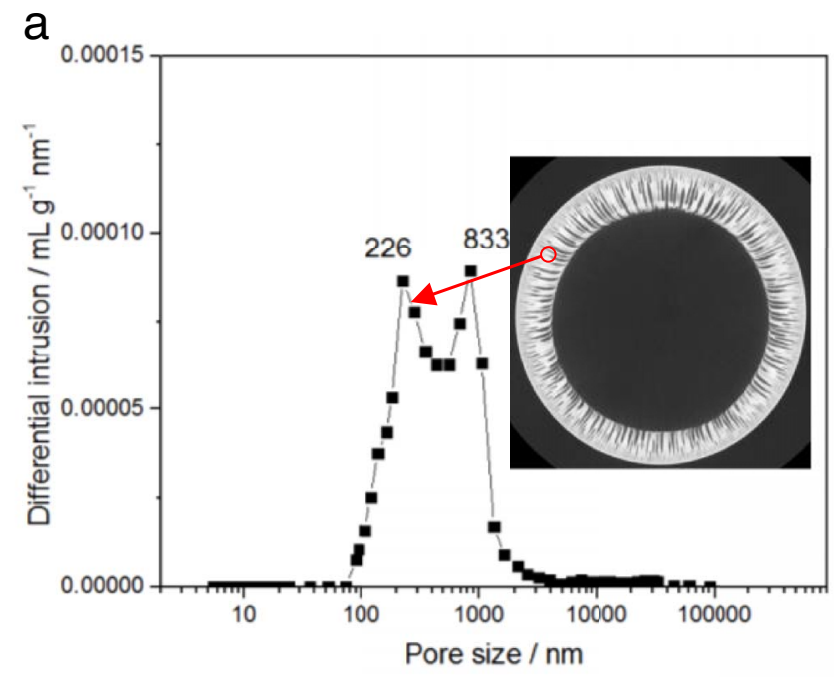

b

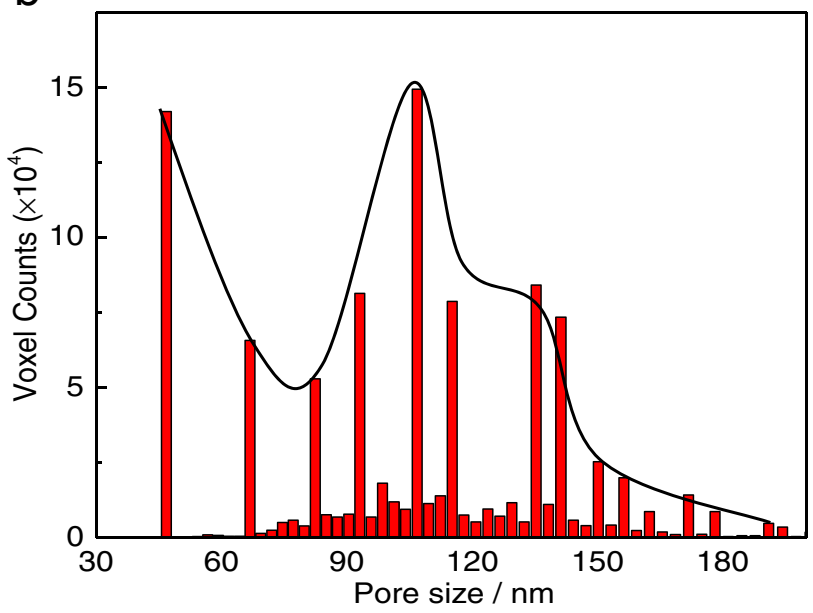

Figure 4. Comparison of the pore diameter measurement using (a) mercury intrusion porosimetry and (b) CT analysis. 

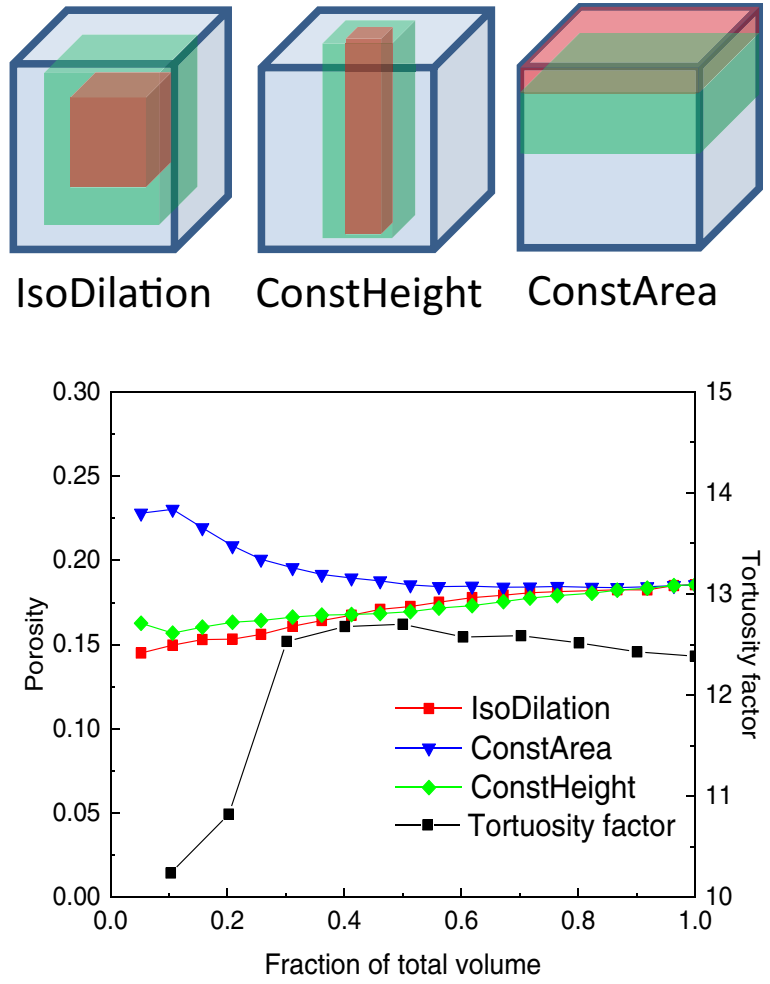

Figure 5. Schematics of the three methods used for RVA and the corresponding results.

Diffusion regime characterizations: $\mathrm{H}_{2} / \mathrm{H}_{2} \mathrm{O}$ and $\mathrm{CH}_{4} / \mathrm{H}_{2} \mathrm{O}$.The computed local pore size of the anode was converted into the 3D distributions of the Knudsen number for the gas species of $\mathrm{H}_{2}$ and $\mathrm{CH}_{4}$, respectively in $\mathrm{H}_{2} / \mathrm{H}_{2} \mathrm{O}$ and $\mathrm{CH}_{4} / \mathrm{H}_{2} \mathrm{O}$ at incremental SOFC operating temperatures (i.e. 600,800 and $1000^{\circ} \mathrm{C}$ ) using Eq. 1 (Fig. 6). The $\mathrm{CH}_{4} / \mathrm{H}_{2} \mathrm{O}$ mixture is used as a model platform to vary the Knudsen number of the diffusing species and thus make a comparison with $\mathrm{H}_{2} / \mathrm{H}_{2} \mathrm{O}$, which shows a different Knudsen number in the same operating conditions. The Knudsen number of $\mathrm{H}_{2}$ is higher $(1.1 \sim 4.8)$ than $\mathrm{CH}_{4}(0.6 \sim 3)$ at all three temperatures because of the larger mean free path, and it is consistent with the earlier work stating the Knudsen number is $0.1<K_{n}<5$ for most of the SOFC electrodes, ${ }^{29}$ which indicates diffusion processes are governed by both molecular and Knudsen mechanisms for both gases here.

The volume matrices, storing the voxel-by-voxel pore size of the anode, were then used to calculate the 3D distribution of Knudsen diffusion coefficient $\left(D_{i}{ }^{k}\right)$ at different temperatures for two gas species using Eq. 5. Given the binary diffusion coefficient $D_{i j}$ which are calculated for $\mathrm{H}_{2} / \mathrm{H}_{2} \mathrm{O}$ and $\mathrm{CH}_{4} / \mathrm{H}_{2} \mathrm{O}$, respectively, at incremental temperatures, the effective diffusion coefficient $\left(D_{\text {eff }}\right)$ was obtained using Bosanquet equation (i.e. Eq. 6), and visualized in 3D (Fig. 7). It shows that the magnitude and range of $D_{\text {eff }}$ are significantly larger and wider for $\mathrm{H}_{2}$ than for $\mathrm{CH}_{4}$ at the three temperatures investigated. This could inevitably cause inaccuracies if measuring the gas diffusion flow assuming a single diffusion coefficient based on an averaged pore size. To estimate the maximum mass transport resistance, the mass flow could be determined by the smallest pore in the diffusion regime, where there is strongest Knudsen effect and can introduce remarkable resistance due to the constrictivity. ${ }^{52}$ In contrast, the Knudsen number for $\mathrm{CH}_{4}$ is lower, hence, a larger fraction of pores lie in the proximity of the continuum regime.

Observing the histogram distribution of $D_{\text {eff }}$ in Fig. 8, the macroscopic $D_{\text {eff }}$ of $\mathrm{H}_{2}$ is approximately two times larger than that of $\mathrm{CH}_{4}$. This is due to the larger $D_{i j}$ of the $\mathrm{H}_{2} / \mathrm{H}_{2} \mathrm{O}$ gas mixture compared to $\mathrm{CH}_{4} / \mathrm{H}_{2} \mathrm{O}$ and the smaller molecular weight of $\mathrm{H}_{2}$ compared to $\mathrm{CH}_{4}$, which makes $D_{\mathrm{H}_{2}}{ }^{k}>D_{\mathrm{CH} 4}{ }^{k}$ (see Eq. 5). The minimum and maximum
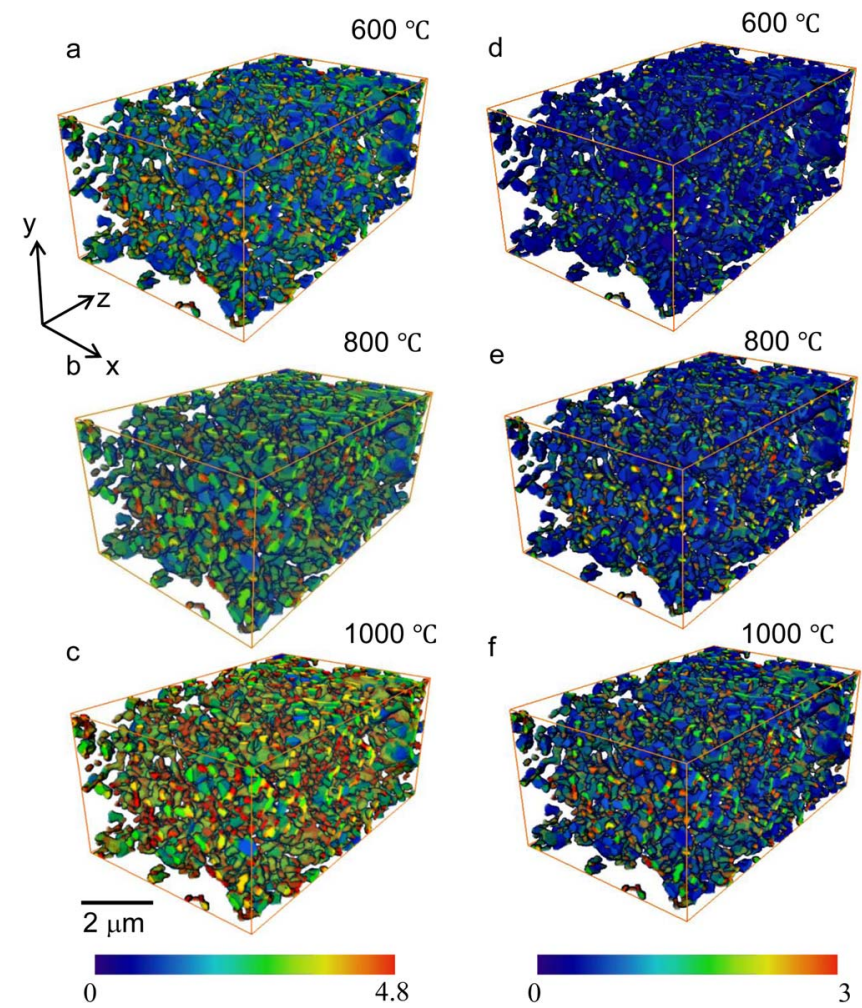

Figure 6. 3D distribution of the Knudsen number in the anode for diffusion gas of $\mathrm{H}_{2}(\mathrm{a}-\mathrm{c})$ and $\mathrm{CH}_{4}$ (d-f) respectively.
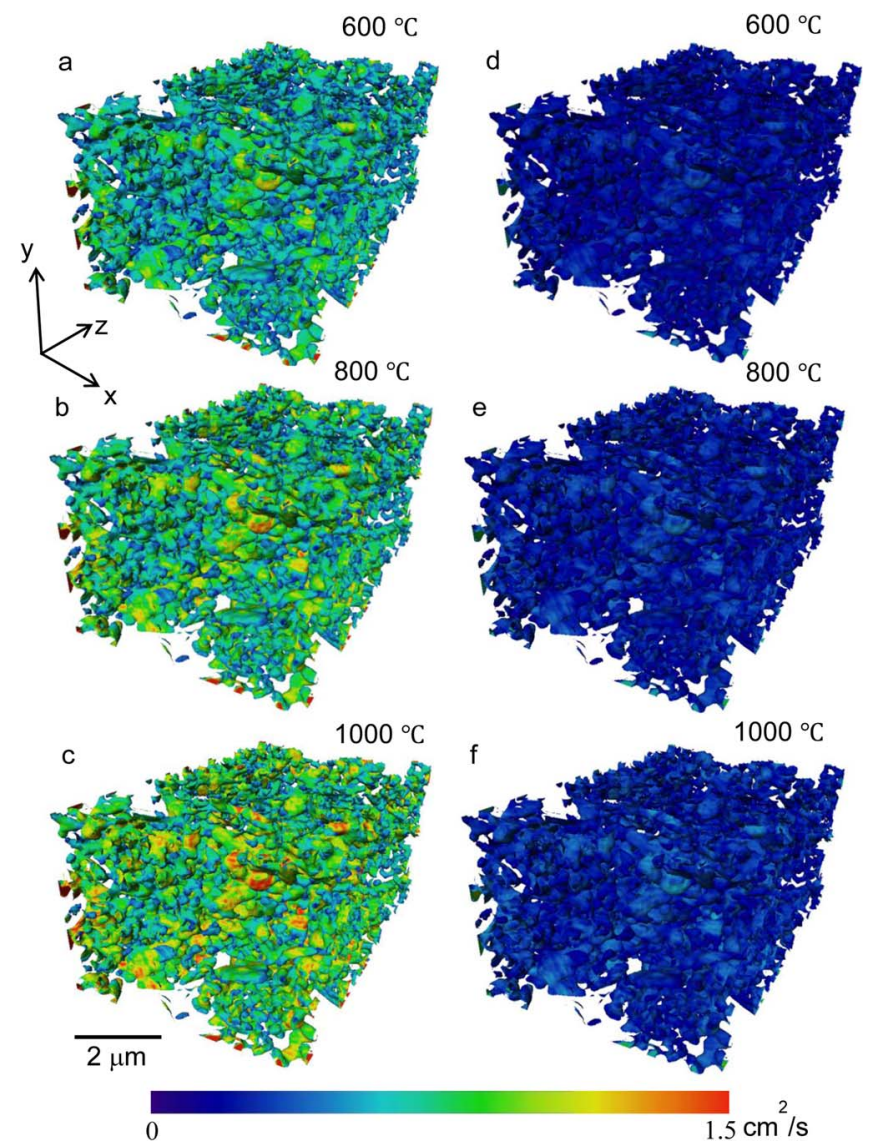

Figure 7. 3D distribution of the effective diffusion coefficient $D_{\text {eff }}$ for $\mathrm{H}_{2}$ (a-c) and $\mathrm{CH}_{4}$ (d-f) in diffusion regimes $\mathrm{H}_{2} / \mathrm{H}_{2} \mathrm{O}$ and $\mathrm{CH}_{4} / \mathrm{H}_{2} \mathrm{O}$ respectively according to the Bosanquet expression Eq. 6. 


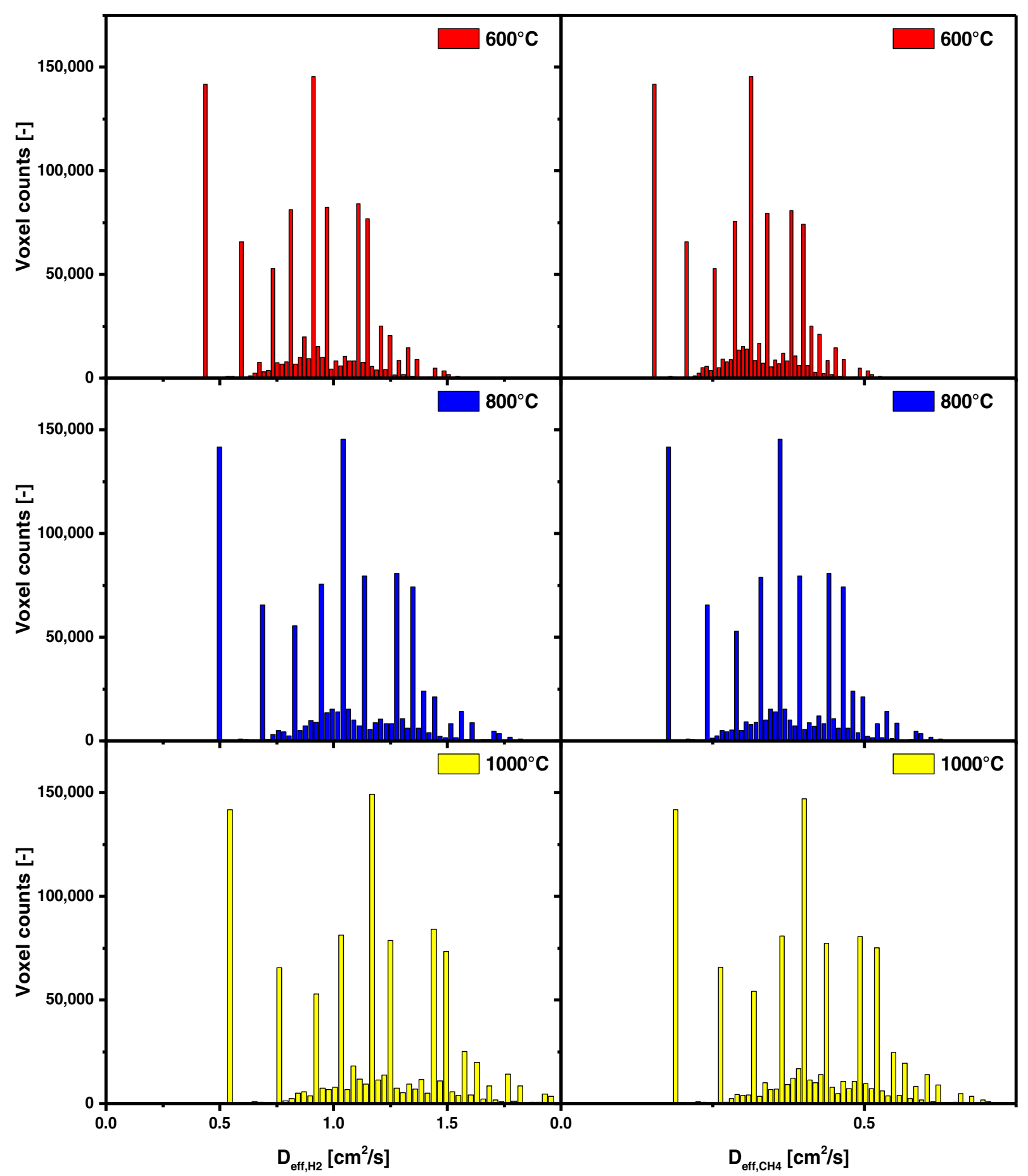

Figure 8. Histogram of effective diffusion coefficient $D_{\text {eff }}$ at incremental temperatures for $\mathrm{H}_{2}$ (left) and $\mathrm{CH}_{4}$ (right) respectively.

$D_{\text {eff }}$ are measured to be 0.5 and $2.5 \mathrm{~cm}^{2} / \mathrm{s}$ for $\mathrm{H}_{2}$ and 0.2 and $0.7 \mathrm{~cm}^{2} / \mathrm{s}$ for $\mathrm{CH}_{4}$, respectively. Seen from Fig. 8, the histograms of $D_{\text {eff }}$ for three temperatures are identical except for the shift in $x$ axis.

It is evident from Table III that $D_{i j}$ is more subject to the variation of the temperature as it increases by $35 \sim 60 \%$ at each incremental $200^{\circ} \mathrm{C}$ compared to $10 \sim 15 \%$ of $D_{i}{ }^{k}$. This is due to the different temperature dependences of the diffusion coefficients: $D_{i j}$ scales with $T^{1.75}$ according to Eq. 4 while $D_{i}{ }^{k}$ scales with $T^{0.5}$ according to Eq. 5. This means that the temperature has a more important role in molecular diffusion regime than under Knudsen diffusion regime.

It is noted that $D_{i j}$ of both $\mathrm{H}_{2}$ and $\mathrm{CH}_{4}$ are $6 \sim 10$ times larger than $D_{i}{ }^{k}$, which suggests that $D_{\text {eff }}$ is heavily affected by $D_{i}{ }^{k}$ rather than
$D_{i j}$ in the transition diffusion regime for this material, though $\mathrm{CH}_{4}$ displays a much lower $K_{n}$. This is because the binary mixture of $\mathrm{CH}_{4}$ and $\mathrm{H}_{2} \mathrm{O}$ contributes to a larger representative mean free path for $\mathrm{CH}_{4}$ compared to that under the self-diffusing condition as the molecular diameter of $\mathrm{CH}_{4}(3.7 \AA)$ is larger than $\mathrm{H}_{2} \mathrm{O}(2.6 \AA)$, consequently amplifying the Knudsen effect. Likewise, the combination of $\mathrm{H}_{2}$ and $\mathrm{H}_{2} \mathrm{O}$ mitigates the Knudsen effect for $\mathrm{H}_{2}$.

Steady-state gas diffusion.- The broad range of $D_{\text {eff }}$ due to local pore size variation, together with the local tortuosity and constrictivity effects can lead to the over/under estimation of the mass flux/flow by using an averaged pore size, especially for an anode operating in 


\begin{tabular}{|c|c|c|c|c|c|c|}
\hline \multicolumn{7}{|c|}{$\begin{array}{l}\text { Table III. Comparisons of } D_{i j} \text {, average } D_{i}{ }^{k} \text { and } D_{e f f} \text { of } \mathrm{H}_{2} \text { and } \mathrm{CH}_{4} \\
\text { in } \mathrm{H}_{2} / \mathrm{H}_{2} \mathrm{O} \text { and } \mathrm{CH}_{4} / \mathrm{H}_{2} \mathrm{O} \text { at incremental temperatures. Considered } \\
d_{p}=0.11 \mu \mathrm{m} \text {, corresponding to volume averaged pore diameter. }\end{array}$} \\
\hline & \multicolumn{2}{|c|}{$D_{i j}\left(\mathrm{~cm}^{2} / \mathrm{s}\right)$} & \multicolumn{2}{|c|}{$D_{i}{ }^{k}\left(\mathrm{~cm}^{2} / \mathrm{s}\right)$} & \multicolumn{2}{|c|}{$D_{e f f}\left(\mathrm{~cm}^{2} / \mathrm{s}\right)$} \\
\hline & $\mathrm{H}_{2}$ & $\mathrm{CH}_{4}$ & $\mathrm{H}_{2}$ & $\mathrm{CH}_{4}$ & $\mathrm{H}_{2}$ & $\mathrm{CH}_{4}$ \\
\hline $600^{\circ} \mathrm{C}$ & 5.78 & 1.75 & 0.97 & 0.34 & 0.83 & 0.28 \\
\hline $800^{\circ} \mathrm{C}$ & 8.29 & 2.52 & 1.11 & 0.40 & 0.97 & 0.34 \\
\hline $1000^{\circ} \mathrm{C}$ & 11 & 3.4 & 1.27 & 0.45 & 1.14 & 0.39 \\
\hline
\end{tabular}

the transition diffusion regime. This may result in inaccuracies in the evaluation of concentration overpotentials and thus, of the global electrochemical performance of SOFC. Based on recent work by the authors, porous phases with same porosity and mean pore size can demonstrate different mass transport properties. Here we assess the variation of the steady-state mass flux of $\mathrm{H}_{2}$ in a binary mixture by Fick's law using three representative $D_{\text {eff }}$ based on the distribution histogram of diffusion gas $\mathrm{H}_{2}$ at $1000^{\circ} \mathrm{C}$ : $\mathrm{LB}\left(0.54 \mathrm{~cm}^{2} / \mathrm{s}\right), \mathrm{AG}(1.14$ $\left.\mathrm{cm}^{2} / \mathrm{s}\right)$ and UB $\left(2.25 \mathrm{~cm}^{2} / \mathrm{s}\right)$.

Results show pronounced differences in terms of mass flux for the three cases (Fig. 9) and a heterogeneous flux distribution is observed for all the three cases. The flux is generally larger at the location where there is a sharp change of pore diameter (i.e. constrictivity). This is more clearly seen in the zoomed in image (Fig. 9d), where the flux vector clearly shows a high flux at the pore neck, where a larger $\mathrm{H}_{2}$ concentration gradient exists. Fig. 10a compares the magnitude of the mass fluxes, averaged on the local cross section area of the porous phase, traversing the cross section planes in the diffusion direction. Note that the local cross section area of the porous phase is not uniform along the diffusion direction. The $\mathrm{H}_{2}$ mass flow for the upper boundary (UB) of $D_{\text {eff }}$ is approximately 2.5 times larger as average (AG) $D_{\text {eff }}$ and 4 times larger as lower boundary (LB) of $D_{\text {eff. }}$ The disparities of the mass flow would result in different concentration polarization behaviors. Although absolute values may change from sample to sample, the emerging trends and the relative ratios are fundamental properties that don't vary. Fig. 10b displays the calculated concentration overpotential $\eta\left(\eta=-R T / 2 F \cdot \ln \left(1-i / i_{L}\right), i\right.$ is current

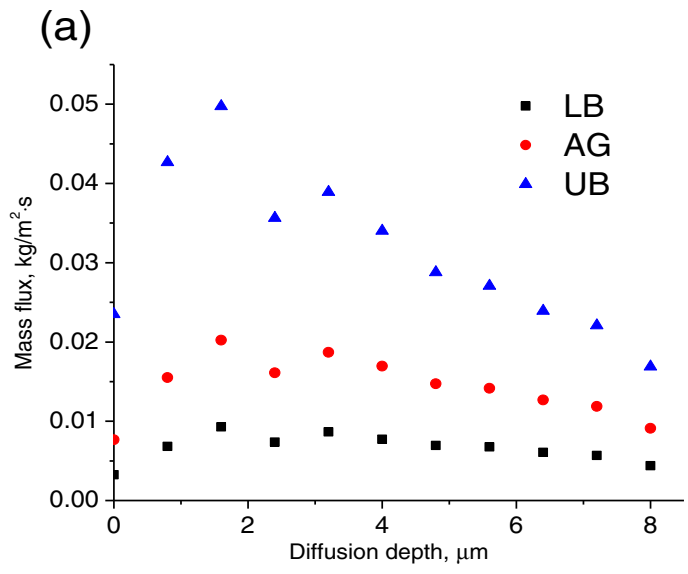

(b)

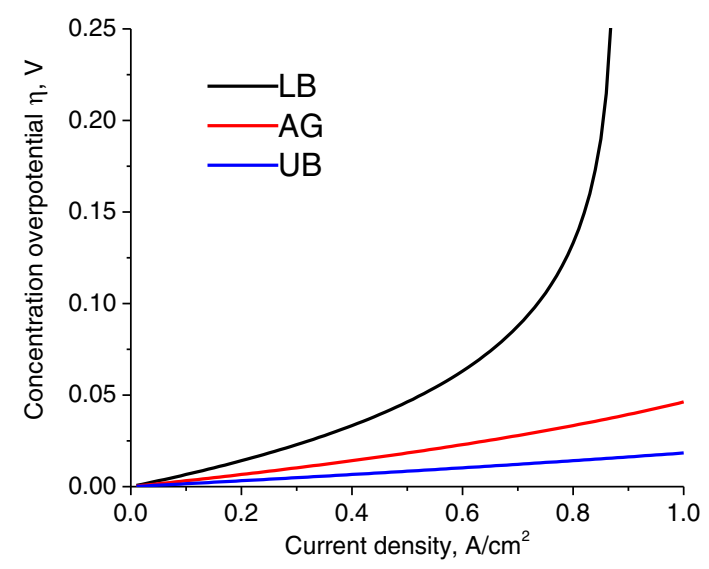

Figure 10. (a) The magnitude of the mass flux of $\mathrm{H}_{2}$ in the diffusion domain are plotted against the diffusion depth away from the initial plane; (b) concentration overpotential comparisons of three diffusion cases for $y_{H 2}{ }^{I N}=1$, $L=400 \mu \mathrm{m}$ and $T=1000^{\circ} \mathrm{C}$. (a)

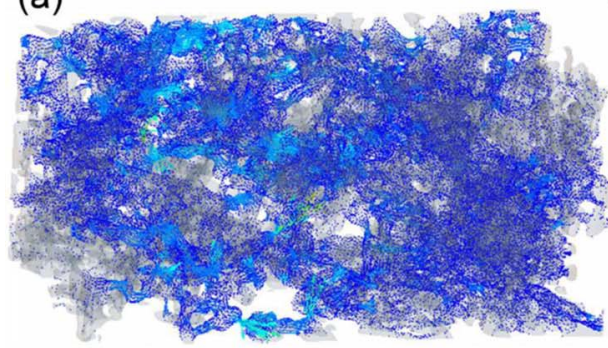

(c)
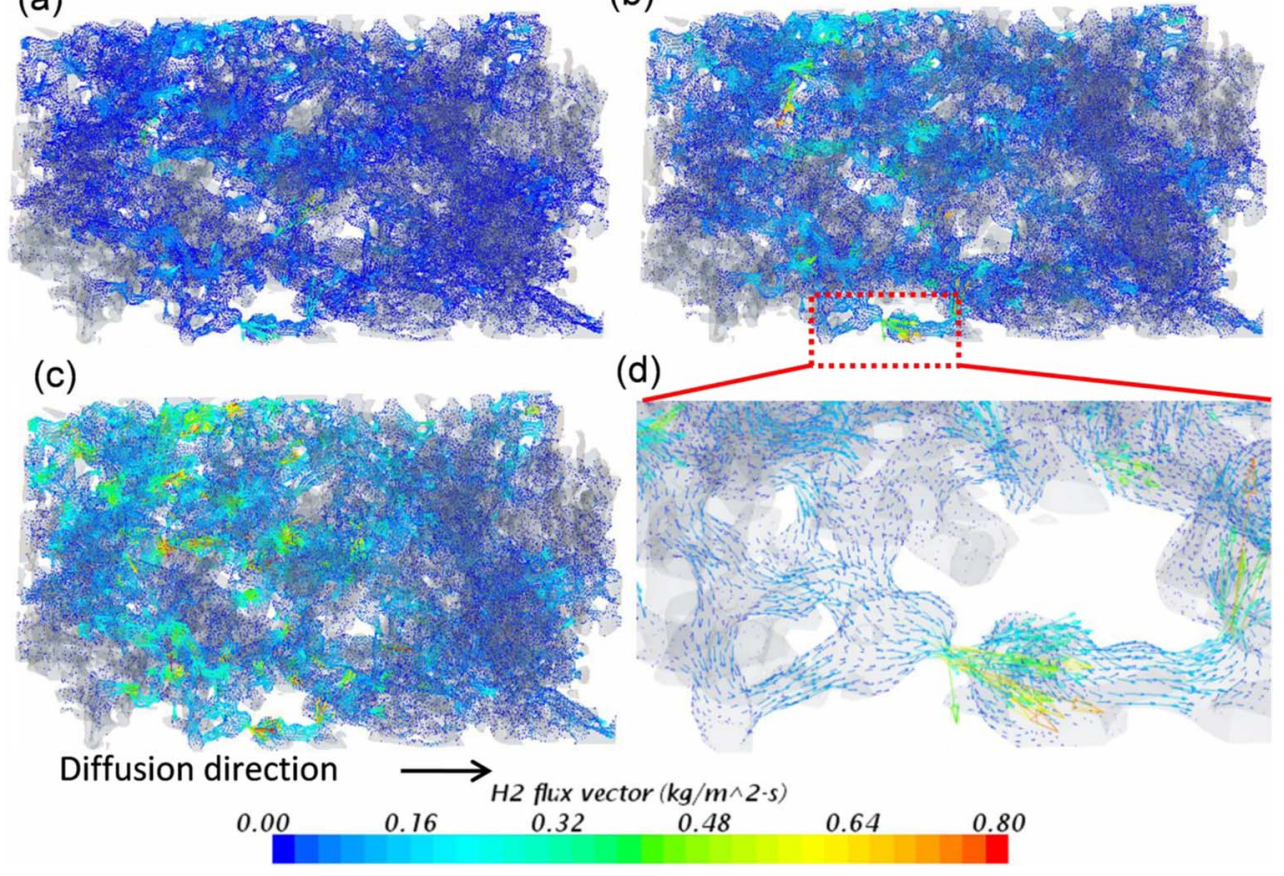

(b)

Figure 9. Mass flux vector of the $\mathrm{H}_{2}$ in the $\mathrm{H}_{2} / \mathrm{H}_{2} \mathrm{O}$ counter diffusion condition with $D_{\text {eff }}$ of $\mathrm{H}_{2}$ at (a) $\mathrm{LB}$, (b) AG, and (c) UB respectively. The local flux vector is shown in (d) showing the constrictivity effect. The gas molecules diffuse from LHS to RHS. 
density, $i_{L}$ is limiting current density) based on three diffusion cases using Fick's law as function of current density $i$ at the anode/electrolyte interface. ${ }^{53}$ The values were calculated for an operating temperature of $1000^{\circ} \mathrm{C}$, an anode thickness of $400 \mu \mathrm{m}$ with an inlet $\mathrm{H}_{2}$ mole fraction $y_{H 2}{ }^{I N}=1$. It is evident that the overpotential is significantly higher under the LB case compared to the remaining two cases. The difference amounts to approximately $50 \%$ between the cases LB and AG. These differences can be more serious when the electrochemical performance is simulated at a larger operating current density.

As discussed above, the broad distribution of pore diameters in the SOFC anode can result in visible variations of mass flux and polarization, if a volume-averaged pore thickness is used to impose the Knudsen diffusion effect on the system. This result is generally valid in the normal range of operation of SOFCs. For more extreme conditions, such as higher Knudsen numbers, results may be affected by the limitations of the modelling approach used. In fact, the Knudsen effect is approximated here using continuum physics solution, and the slip at the interior walls of the pore is neglected, which can be significant for high $K_{n}$ diffusion case. In order to more sensibly estimate the mass transport in the transition region using continuum physics, the representative pore size can be determined using an experimental method by comparing the flux ratio of two gas species with varying pressure gradient and compare it with that predicted by continuum physics solution. $^{12}$

\section{Conclusions}

Mass transport in SOFC anodes is critical in determining the reaction rate and electrochemical performance. Here, we have investigated the pore size distribution of an SOFC anode fabricated by phase-inversion technique via X-ray nano-CT, which enables the pore size measurement in 3D at sub-100 nm length scales. For the anode studied, a broad range of pore size distribution $(32 \sim 600 \mathrm{~nm})$ was observed, resulting in a Knudsen number distribution between 1.1 to 4.8 and 0.6 to 3 for $\mathrm{H}_{2}$ and $\mathrm{CH}_{4}$, respectively at an operating temperature of $1000^{\circ} \mathrm{C}$. This warrants diffusion under the transition regime, which consists of both molecular and Knudsen diffusion mechanisms, in the anode for both fuel gases. However, $\mathrm{CH}_{4}$ will result in a diffusion flux 4 times lower than that given by $\mathrm{H}_{2}$. In continuum physics, a representative averaged pore size should be chosen experimentally: by measuring the molar flux ratio of two gas species at varying pressure gradient at inlet/outlet and a representative pore diameter can be determined based on which the model prediction matches the experiment results. Alternatively, a numerical simulation which can accommodate the distribution of $D_{\text {eff }}$ should be undertaken, since it can result in as large as $\pm 200 \%$ error in terms of mass flux, depending on which pore size is chosen in the distribution. In turn, this significantly affects the estimation of the limiting current density and further the concentration overpotential.

\section{Acknowledgments}

The authors acknowledge the support from the EPSRC under grants EP/N032888/1 and EP/M014045/1, PRS acknowledges funding from the Royal Academy of Engineering. Dr. Antonio Bertei acknowledges funding from the European Union's Horizon 2020 research and innovation programme under the Marie Skłodowska-Curie grant agreement No 654915.

\section{References}

1. A. J. Bard, L. R. Faulkner, J. Leddy, and C. G. Zoski, Electrochemical methods: fundamentals and applications, Wiley New York (1980).

2. R. G. Carbonell and S. Whitaker, in Fundamentals of Transport Phenomena in Porous Media, p. 121, Springer, (1984).

3. K. Vafai, Handbook of Porous Media, Crc Press (2015).

4. W. He, J. Zou, B. Wang, S. Vilayurganapathy, M. Zhou, X. Lin, K. H. Zhang, J. Lin, P. Xu, and J. H. Dickerson, Journal of Power Sources, 237, 64 (2013).
5. A. Kromp, H. Geisler, A. Weber, and E. Ivers-Tiffée, Electrochimica Acta, 106, 418 (2013).

6. Y. Fu, Y. Jiang, S. Poizeau, A. Dutta, A. Mohanram, J. D. Pietras, and M. Z. Bazant, Journal of The Electrochemical Society, 162(6), F613 (2015).

7. A. Bertei, B. Nucci, and C. Nicolella, Chemical Engineering Science, 101, 175 (2013).

8. W. Lehnert, J. Meusinger, and F. Thom, Journal of Power Sources, 87(1), 57 (2000).

9. P. Shearing, Q. Cai, J. Golbert, V. Yufit, C. Adjiman, and N. Brandon, Journal of Power Sources, 195(15), 4804 (2010).

10. Z. Zhang, J. Chen, D. Yue, G. Yang, S. Ye, C. He, W. Wang, J. Yuan, and N. Huang, Energies, 7(1), 80 (2013)

11. J. Larminie, A. Dicks, and M. S. McDonald, Fuel Cell Systems Explained, Wiley New York (2003).

12. J. Young and B. Todd, International Journal of Heat and Mass Transfer, 48(25), 5338 (2005).

13. B. Tjaden, J. Lane, P. J. Withers, R. S. Bradley, D. J. Brett, and P. R. Shearing, Solid State Ionics, 288, 315 (2016).

14. P. Schneider and J. Smith, AIChE Journal, 14(5), 762 (1968).

15. P. G. Gray and D. D. Do, AIChE journal, 37(7), 1027 (1991).

16. J. Veldsink, R. Van Damme, G. Versteeg, and W. Van Swaaij, The Chemical Engineering Journal and the Biochemical Engineering Journal, 57(2), 115 (1995)

17. R. Ziel, A. Haus, and A. Tulke, Journal of Membrane Science, 323(2), 241 (2008).

18. T. Ferrari, A. Bertei, and C. Nicolella, ECS Transactions, 68(1), 2991 (2015).

19. A. Hernández, J. Calvo, P. Prádanos, and F. Tejerina, Journal of Membrane Science, 112(1), 1 (1996).

20. F. Zhao, T. J. Armstrong, and A. V. Virkar, Journal of the Electrochemical Society, 150(3), A249 (2003)

21. L. Salvo, M. Suéry, A. Marmottant, N. Limodin, and D. Bernard, Comptes Rendus Physique, 11(9), 641 (2010).

22. E. Maire and P. Withers, International Materials Reviews, 59(1), 1 (2014).

23. P. Shearing, J. Golbert, R. Chater, and N. Brandon, Chemical Engineering Science, 64(17), 3928 (2009)

24. P. Shearing, N. Brandon, J. Gelb, R. Bradley, P. Withers, A. Marquis, S. Cooper, and S. Harris, Journal of The Electrochemical Society, 159(7), A1023 (2012).

25. S. Cooper, D. Eastwood, J. Gelb, G. Damblanc, D. Brett, R. Bradley, P. Withers, P. Lee, A. Marquis, and N. Brandon, Journal of Power Sources, 247, 1033 (2014).

26. D. Kehrwald, P. R. Shearing, N. P. Brandon, P. K. Sinha, and S. J. Harris, Journal of The Electrochemical Society, 158(12), A1393 (2011).

27. S. J. Cooper, M. Kishimoto, F. Tariq, R. S. Bradley, A. J. Marquis, N. P. Brandon, J. A. Kilner, and P. R. Shearing, ECS Transactions, 57(1), 2671 (2013).

28. J. Yuan and B. Sundén, International Journal of Heat and Mass Transfer, 69, 358 (2014).

29. W. He, W. Lv, and J. Dickerson, Gas Transport in Solid Oxide Fuel Cells, Springer (2014).

30. O. O. Taiwo, D. P. Finegan, D. S. Eastwood, J. L. Fife, L. D. Brown, J. A. Darr P. D. Lee, D. J. Brett, and P. R. Shearing, Journal of Microscopy, 263(3), 280 (2016).

31. B. Münch and L. Holzer, Journal of the American Ceramic Society, 91(12), 4059 (2008).

32. R. G. Mortimer, Mathematics for Physical Chemistry, Academic Press (1999).

33. T. Fiedler, I. Belova, and G. Murch, Computational Materials Science, 50(5), 1795 (2011).

34. I. Kinefuchi, J. Oyama, K. Yokoyama, N. Kubo, T. Tokumasu, and Y. Matsumoto, ECS Transactions, 58(1), 71 (2013).

35. A. Kalarakis, V. Michalis, E. Skouras, and V. Burganos, Transport in Porous Media 94(1), 385 (2012).

36. R. B. Bird, Applied Mechanics Reviews, 55(1), R1 (2002)

37. A. Berson, H.-W. Choi, and J. G. Pharoah, Physical Review E, 83(2), 026310 (2011)

38. J. Laurencin, R. Quey, G. Delette, H. Suhonen, P. Cloetens, and P. Bleuet, Journal of Power Sources, 198, 182 (2012).

39. E. N. Fuller, P. D. Schettler, and J. C. Giddings, Industrial \& Engineering Chemistry, 58(5), 18 (1966)

40. B. Haberman, C. M. Baca, and T. Ohrn, ECS Transactions, 35(1), 451 (2011)

41. W. G. Pollard and R. D. Present, Physical Review, 73(7), 762 (1948).

42. R. Krishna and J. M. van Baten, Chemical Engineering Science, 69(1), 684 (2012).

43. S. M. Jamil, M. H. D. Othman, M. A. Rahman, J. Jaafar, A. F. Ismail, and K. Li, Journal of the European Ceramic Society, 35(1), 1 (2015).

44. K. Li, Ceramic membranes for separation and reaction John Wiley \& Sons Ltd, Chichester, UK (2007).

45. T. Li, Z. Wu, and K. Li, Journal of Power Sources, 273, 999 (2015).

46. H. Strathmann and K. Kock, Desalination, 21(3), 241 (1977).

47. S. Liu, Ceramics International, 29(8), 875 (2003)

48. T. Li, PhD thesis, Imperial College London, 78 (2015).

49. R. Dougherty and K.-H. Kunzelmann, Microscopy and Microanalysis, 13(S02), 1678 (2007).

50. M. Doube, M. M. Kłosowski, I. Arganda-Carreras, F. P. Cordelières, R. P. Dougherty, J. S. Jackson, B. Schmid, J. R. Hutchinson, and S. J. Shefelbine, Bone, 47(6), 1076 (2010).

51. S. J. Cooper, A. Bertei, P. R. Shearing, J. A. Kilner, and N. P. Brandon, SoftwareX, 5, $203(2016)$

52. L. Holzer, D. Wiedenmann, B. Münch, L. Keller, M. Prestat, P. Gasser, I. Robertson, and B. Grobéty, Journal of Materials Science, 48(7), 2934 (2013).

53. U. Doraswami, P. Shearing, N. Droushiotis, K. Li, N. P. Brandon, and G. H. Kelsall, Solid State Ionics, 192(1), 494 (2011). 